\title{
Ground-state properties of weakly bound helium-alkali trimers
}

P. Stipanović, ${ }^{1}$ L. Vranješ Markić, ${ }^{1}$ D. Zarić, ${ }^{2}$ and J. Boronat ${ }^{3}$

1) Faculty of Science, University of Split, Ruđera Boškovića 33, HR-21000 Split, Croatia ${ }^{\mathrm{a})}$

${ }^{2)}$ Faculty of Electrical Engineering, Mechanical Engineering and Naval Architecture, University of Split, R. Boškovića 32, HR-21000 Split, Croatia

3) Departament de Física, Campus Nord B4-B5, Universitat Politècnica de Catalunya, E-08034 Barcelona, Spain

(Dated: 12 December 2016)

Weakly bound triatomic molecules consisting of two helium atoms and one alkali metal atom are studied by means of the diffusion Monte Carlo method. We determined the stability of ${ }^{4} \mathrm{He}_{2} \mathrm{~A},{ }^{4} \mathrm{He}^{3} \mathrm{HeA}$ and ${ }^{3} \mathrm{He}_{2} \mathrm{~A}$, where $\mathrm{A}$ is one of the alkali atoms $\mathrm{Li}, \mathrm{Na}, \mathrm{K}, \mathrm{Rb}$ or Cs. Some of the trimers with ${ }^{3} \mathrm{He}$ are predicted to be self-bound for the first time, but this is observed to be dependent on the He-A interaction potential model. In addition to the ground-state energy of the trimers, we determined their density, radial and angular distributions. Many of them are spatially very extended, which qualifies them as quantum halo states.

PACS numbers: 02.70.Ss, 36.40.-c, 67.90. $+\mathrm{z}$

a) pero@pmfst.hr 


\section{INTRODUCTION}

Weakly bound and spatially extended few-particle systems are of interest in many branches of physics, from ultracold gases to nuclear physics. Many of them can be classified as quantum halo states, which are usually defined as bound states with a probability higher than $50 \%$ of occupying the classically forbidden region. ${ }^{1,2}$ The criterion is most precisely verified by calculating their scaled size. ${ }^{1,2}$ Some also exhibit Efimov states. ${ }^{3}$ The latter present an infinite series of loosely bound three-body states, which appears when the two-body subsystem has a zero-energy ground state. There are several molecular systems that share quantum halo characteristics, the most notable of them being helium trimers, ${ }^{4} \mathrm{He}_{3}$ and ${ }^{4} \mathrm{He}_{2}{ }^{3} \mathrm{He}$. The excited state of ${ }^{4} \mathrm{He}_{3}$ has recently been confirmed ${ }^{4}$ as an Efimov state.

Diffuseness in few-body clusters is intimately related to the strength of the van der Waals attraction between their constituents and their mass. The pair interaction between He and an alkali atom is even shallower than the He-He interaction, so that the formed pairs are very diffuse $^{5}$ and thus neat candidates for quantum halo states. The energy of the ${ }^{4} \mathrm{He}-{ }^{7} \mathrm{Li}$ dimer was predicted to be $-5.6 \mathrm{mK}$, with a mean interparticle distance $<r>=28 \AA$. Recently, this dimer was experimentally detected ${ }^{6}$.

Very weak binding has been predicted for trimers containing two helium and one alkali atom. Particularly, mixtures of ${ }^{4} \mathrm{He}_{2} \mathrm{~A}$, where $\mathrm{A}$ is one of the alkali atoms, were theoretically investigated using different methods ${ }^{7-16}$. An Efimov state was also theoretically predicted for the first excited state of ${ }^{4} \mathrm{He}_{2}{ }^{6} \mathrm{Li}$ and ${ }^{4} \mathrm{He}_{2}{ }^{7} \mathrm{Li}$. The results differed depending on the interaction potential model and the theoretical method used for the study. Trimers ${ }^{3} \mathrm{He}^{4} \mathrm{HeA}$ and ${ }^{3} \mathrm{He}_{2} \mathrm{~A}$ have been scarcely characterized. Yuan and $\operatorname{Lin}^{7}$ predicted that some of the trimers are bound with $\mathrm{A}={ }^{6} \mathrm{Li},{ }^{7} \mathrm{Li}$ and ${ }^{23} \mathrm{Na}$ using old forms of the interaction potentials, while upper and lower energy ranges for $\mathrm{A}={ }^{39} \mathrm{~K},{ }^{85} \mathrm{Rb}$ were given in Refs. 12 and 14 respectively. Furthermore, in a recent work on universality of quantum halo trimers ${ }^{17}$ we have calculated the ground-state properties of the trimers, ${ }^{3} \mathrm{He}^{4} \mathrm{He}^{7} \mathrm{Li},{ }^{3} \mathrm{He}^{4} \mathrm{He}^{39} \mathrm{~K},{ }^{3} \mathrm{He}^{4} \mathrm{He}^{41} \mathrm{~K}$, ${ }^{3} \mathrm{He}_{2}{ }^{23} \mathrm{Na},{ }^{3} \mathrm{He}_{2}{ }^{41} \mathrm{~K}$, and ${ }^{3} \mathrm{He}_{2}{ }^{85} \mathrm{Rb}$. In all of these quoted works the analysis of the structure of ${ }^{3} \mathrm{He}^{4} \mathrm{HeA}$ and ${ }^{3} \mathrm{He}_{2} \mathrm{~A}$ clusters has not been done. At the same time, since He-A trimers with ${ }^{3} \mathrm{He}$ are predicted to be even more weakly bound, it is expected that they will be even more delocalized than the ${ }^{4} \mathrm{He}_{2} \mathrm{~A}$ trimers. It would be very interesting to investigate if their shapes differ substantially from the shapes of the ${ }^{4} \mathrm{He}_{2} \mathrm{~A}$ clusters and how it changes with 
the mass of the alkali atom.

Furthermore the distributions of interparticle distances, as well as the angular distributions, of the ${ }^{4} \mathrm{He}_{3}$ and ${ }^{4} \mathrm{He}_{2}{ }^{3} \mathrm{He}$ trimers have been recently measured. ${ }^{4,18}$ This opens up the possibility that He-A trimers could be measured in a similar setting. This would enable also the testing of the He-A interaction potentials, which are not as well known as the He-He interactions. Namely, for the helium trimers, we have recently shown ${ }^{19}$ that measured distribution functions can be used to rate the quality of the He-He interaction potentials. If available, such information would be valuable for studies of alkali atoms interacting with nanoscopic helium droplets.

In this work, we report ground-state properties of He-A trimers using quantum Monte Carlo. Our aim has been to determine with accuracy the energy and radial and angular distributions of a wide collection of trimers. We include results for ${ }^{4} \mathrm{He}$, but also for ${ }^{3} \mathrm{He}$ clusters, which have been less studied in the past. The influence of the He-A model potential in the results is also analyzed in depth. The very weak binding energies and spatial extent observed in these clusters, mainly when ${ }^{3} \mathrm{He}$ is present, suggests the appearance of new molecular halo states.

The rest of the paper is organized as follows. In Sec. II, we introduce the selected potential models and the diffusion Monte Carlo method (DMC) ${ }^{20}$. We also discuss the trial wave functions used for importance sampling. Sec. III reports the energies and distribution functions for the trimers under study. Finally, Sec. IV comprises a summary of the work and an account of the main conclusions.

\section{METHOD}

The DMC method provides exact results for the ground-state properties of the trimers under study once the models for the different pair interactions are chosen. In the following, we describe first the pair potentials used in our work and next we briefly describe the DMC method including pure estimators for an accurate calculation of the distribution properties. ${ }^{21}$ 


\section{A. Interaction potential models}

We modeled the interaction of He atoms with the semi-empirical HFDB form given by Aziz et al. ${ }^{22}$ who adapted the B-type of Hartree-Fock model with damped dispersion (HFD) to experimental and theoretical results. In a recent study of helium trimers ${ }^{19}$ it has been shown that it gives results very close to the most sophisticated theoretical $V_{\mathrm{BO}}$-model, published few years ago by Jeziorska et $a .^{23}$. The He-He potential does not depend on the particular He isotope. For the interaction of He atoms with alkali ones, we have used four different models. The model labeled KTTY comes from the work by Kleinekathöfer, Tang, Tonnies and Yiu, who calculated He-A interactions using the surface integral model. ${ }^{24}$ Since the original form was mathematically complicated and impractical, in a subsequent work Kleinkathöfer, Lewerenz and Mladenović ${ }^{5}$ recasted the original potential to a simpler modified Tang-Toennies form by means of a least-squares fit. In the process, they also chose the newer version of the He-Li dispersion coefficients ${ }^{25}$. In the present work, we have thus chosen the latter, more practical form ${ }^{5}$ as the KTTY model. For some systems we also use additional models. First is the latest available XKMX model by Xie et al. ${ }^{26}$. It is an analytical pair potential model, whose parameters were obtained as a fit to the original KTTY data. Next model, PSL, available only for Li, Na and K, is by Partrige et al. ${ }^{27}$, who have used $a \mathrm{~b}$ initio energies to construct potential data and connected them to an older form of the dispersion coefficients ${ }^{28}$. The last model that we use for comparison is the older one by Cvetko. ${ }^{29}$ The He-A interaction potentials are shown in Fig. 1. Their hard core increases from Li to Cs and is substantially larger than in the case of He-He potentials (not shown), where it is $\sim 2.6 \AA$. He-A potentials are also much shallower than He-He one, whose depth is about $-11 \mathrm{~K}$. Our calculations rely on the KTTY model, the other potentials being only used for comparison in the selected cases. In Fig. 1 (a), comparison with Cvetko model is presented only in the He-K case, where it is slightly deeper than KTTY model. In fact, it is also deeper in the case of $\mathrm{Rb}$ and $\mathrm{Cs}$, almost the same for $\mathrm{Na}$ and shallower than KTTY for Li. XKMX and PSL potentials are shown in Fig. 1 (b) only for He-Li and He-Na for comparison. Although XKMX was fitted to the KTTY data, slight shift of the potential well is noticeable, extending the repulsive region of a space. On the other hand PSL is significantly deeper and has a smaller core diameter than others. The other models available in the literature are mainly in between the ones studied here. 


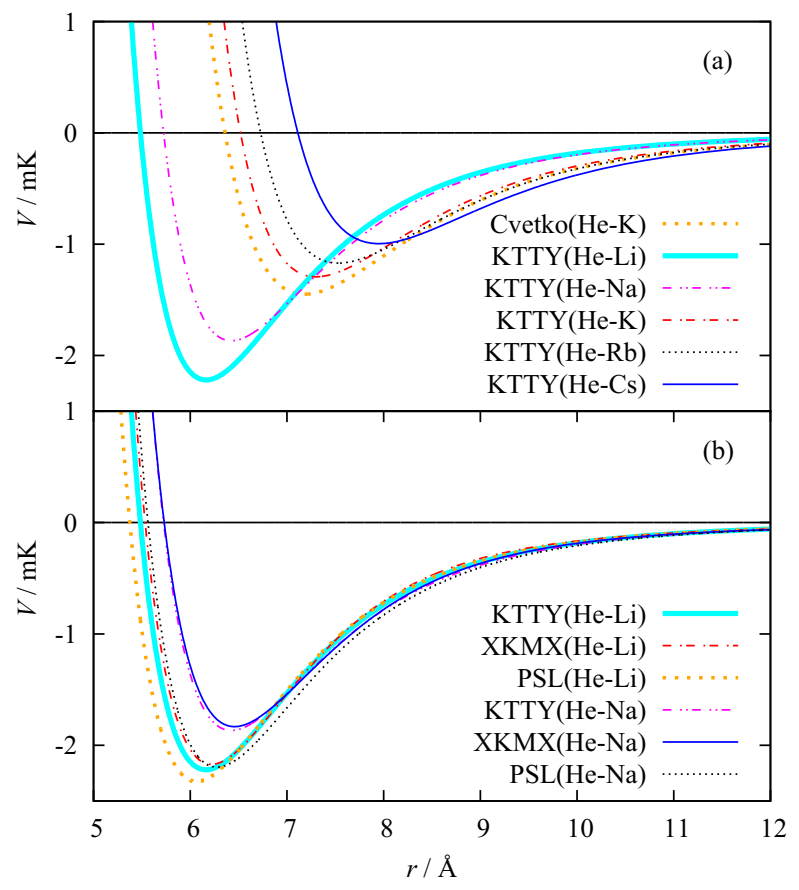

FIG. 1. KTTY ${ }^{5}$ pair potential models $V$ are shown as a function of the separation $r$ between the helium and alkali atoms. Short-range strongly repulsive part and long-range weakly attractive parts are not shown. In panels (a) He-K Cvetko ${ }^{29}$ and (b) He-Li and He-Na PSL ${ }^{27}$ and HKMX ${ }^{26}$ models are added for comparison.

\section{B. Diffusion Monte Carlo method}

As commented previously, in our study we use the DMC method. DMC solves, using a stochastic approach, the Schrödinger equation written in imaginary time $\tau=i t / \hbar$,

$$
-\frac{\partial \Psi(\boldsymbol{R}, \tau)}{\partial \tau}=\left(H-E_{\mathrm{r}}\right) \Psi(\boldsymbol{R}, \tau)
$$

where $E_{\mathrm{r}}$ is a constant acting as a reference energy and $\boldsymbol{R} \equiv\left(\boldsymbol{r}_{1}, \boldsymbol{r}_{2}, \boldsymbol{r}_{3}\right)$ collectively denotes the positions of the trimer constituents. The Hamiltonian $H$ for the helium trimer is

$$
H=-\sum_{i=1}^{3} \frac{\hbar^{2}}{2 m_{i}} \nabla_{i}^{2}+\sum_{\substack{i, j=1 \\ i<j}}^{3} V\left(r_{i j}\right)
$$

where $V$ denotes the interatomic potentials between the three pairs of the trimer. Explicit three-body potentials are not included because their contribution has proved to be negligible in the case of pure He trimers ${ }^{19,30}$. Roudnev and Cavagnero ${ }^{33}$ stressed the sensitivity of benchmarked dimer and trimer properties to fundamental constants. Thus, we used the 
best available data from the NIST database, with constant $0.5 \hbar^{2} m^{-1} /\left(\mathrm{mK} \AA^{2}\right)$ for ${ }^{3} \mathrm{He},{ }^{4} \mathrm{He}$, ${ }^{6} \mathrm{Li},{ }^{7} \mathrm{Li},{ }^{23} \mathrm{Na},{ }^{39} \mathrm{~K},{ }^{41} \mathrm{~K},{ }^{85} \mathrm{Rb}$, and ${ }^{133} \mathrm{Cs}$, respectively equal to 8041.811058, 6059.640786, 4032.226566, 3457.001455, 1055.005713, 622.4853844, 592.1205288, 285.6415818, and 182.4931752.

In order to reduce the variance of the calculation to a manageable level, a common practice is to use importance sampling by introducing a guiding wave function $\psi(\mathbf{R})$. Specifically, the Schrödinger equation (1) is rewritten for the mixed distribution $\Phi(\boldsymbol{R}, \tau)=\Psi(\boldsymbol{R}, \tau) \psi(\boldsymbol{R})$. Within the Monte Carlo framework, $\Phi(\boldsymbol{R}, \tau)$ is represented by a set of walkers $\{\boldsymbol{R}\}$. In the limit $\tau \rightarrow \infty$, for long simulation times, only the lowest energy eigenfunction survives, $\Psi(\boldsymbol{R}, \tau) \rightarrow \psi_{0}(\boldsymbol{R})$ provided certain conditions are met, i.e., $\psi(\mathbf{R})$ must not be orthogonal to the exact ground-state wave function $\psi_{0}(\boldsymbol{R})$ and needs to have non-zero overlap with $\psi_{0}(\boldsymbol{R})$ in all regions where $\psi_{0}(\boldsymbol{R}) \neq 0$. With these conditions, apart from statistical uncertainties, the ground-state energy $E$ of an $N$-body bosonic system is exactly calculated. This applies also to the calculations in the present work because they involve no more than two fermions and thus it is possible to construct trial wave functions without nodes.

To guide the diffusion process, we used trial wave functions which we optimized with the variational Monte Carlo (VMC) method, minimizing the energy and its variance. The trial wave function is of Jastrow type, $\psi\left({ }^{i} \mathrm{He}{ }^{j} \mathrm{He}^{k} \mathrm{~A}\right)=F_{i j}\left(r_{12}\right) F_{i k}\left(r_{13}\right) F_{j k}\left(r_{23}\right)$, i.e., it is formed as a product of two-body correlation functions

$$
F_{m}(r)=\frac{1}{r} \exp \left[-\left(\frac{\alpha_{m}}{r}\right)^{\gamma_{m}}-s_{m} r\right]
$$

In the previous expression, $r$ is the interparticle distance and $m$ stands for a particular pair denoted by atomic numbers $i, j, k$. Variational parameters $\alpha_{m}$ and $\gamma_{m}$ describe short-range correlations, while $s_{m}$ is used for the long-range ones. The optimization of the trial wave functions was done for all clusters and all interaction potential combinations. In the case of the KTTY model for the ${ }^{4} \mathrm{He}_{2} \mathrm{~A}$ cluster, parameter $\alpha_{44}$ was close to $2.77 \AA, \gamma_{44} \approx 4.2$ and $s_{44}$ ranged from 0.001 to $0.02 \AA^{-1}$. In the ${ }^{4} \mathrm{He}-\mathrm{A}$ pair, going from ${ }^{6} \mathrm{Li}$ to ${ }^{133} \mathrm{Cs}, \alpha_{4 A}$ changed from 5.8 to $7.4 \AA$ respectively, $\gamma_{4 A}$ took values from 4.4 to 4.9 and $s_{4 A}$ from 0.03 to $0.001 \AA^{-1}$. The VMC energy was $89 \%-95 \%$ of the DMC one, for all such trimers except for ${ }^{4} \mathrm{He}_{2}{ }^{133} \mathrm{Cs}$, where it was only $45 \%$. The difference between VMC and DMC results increased when one ${ }^{4} \mathrm{He}$ was swapped by ${ }^{3} \mathrm{He}$, and for the cases of ${ }^{3} \mathrm{He}^{4} \mathrm{He}^{6} \mathrm{Li}$ and ${ }^{3} \mathrm{He}^{4} \mathrm{He}^{133} \mathrm{Cs}$ it was not possible to reach binding at the VMC level. Optimal parameters in this case ranged from 2.83 to $2.97 \AA$ for $\alpha_{43}, \gamma_{43}$ from 3.3 to 3.8 and $s_{43}$ from $5 \times 10^{-5}$ to $8 \times 10^{-4} \AA^{-1}$, 
while the parameters for ${ }^{4} \mathrm{He}-\mathrm{A}$ and ${ }^{3} \mathrm{He}-\mathrm{A}$ were in the range previously stated for He- $\mathrm{A}$ interaction in the ${ }^{4} \mathrm{He}_{2} \mathrm{~A}$ trimers. The same set of variational parameters was also obtained in the case of ${ }^{3} \mathrm{He}_{2} \mathrm{~A}$ clusters. The quality of the VMC again dropped and binding at the VMC level was obtained only for ${ }^{3} \mathrm{He}_{2}{ }^{85} \mathrm{Rb}$ and the Cvetko potential.

We used a DMC method ${ }^{20}$ which is accurate to second order in the time step $\Delta \tau$, $E_{\mathrm{DMC}}(\Delta \tau)=E+k_{E}(\Delta \tau)^{2}$. Both the time step dependence and the mean walker population were studied carefully in order to eliminate any bias. For all trimers, 5000 walkers proved to be enough. The DMC energies $E_{\mathrm{DMC}}(\Delta \tau)$ were calculated for different time steps (from $4 \times 10^{-4} \mathrm{~K}^{-1}$ to $16 \times 10^{-4} \mathrm{~K}^{-1}$ ) and the final results were derived by extrapolation to zero time step. It is worth noticing that trimers with ${ }^{3} \mathrm{He}$ atoms were studied with special care due to the above mentioned poor quality of the trial wave function. We carried out additional tests to verify the consistency of the DMC energies in front of significant changes of the variational parameters.

We calculated expectation values of operators which do not commute with the Hamiltonian $H$ using pure estimators. ${ }^{21}$ In this algorithm it is crucial to verify that the chosen block size is large enough to guarantee asymptotic offspring, i.e., to correct the bias coming from the choice of the trial wave function. All presented results for the density profiles $\rho(r)$, the pair $P(r)$ and angular distribution $P(\vartheta)$ functions were obtained using 90000 steps per block, although some properties converged even for 3 times smaller block sizes.

\section{RESULTS}

\section{A. Binding energies}

Our calculated energies for four He-A potential models are presented in Table I in columns entitled with the acronym of the potential and compared to results from other authors, obtained using different methods. The statistical errors are given in parenthesis, e.g., $E\left({ }^{4} \mathrm{He}_{2}{ }^{6} \mathrm{Li}\right)=58.7(2) \mathrm{mK}=58.7 \pm 0.2 \mathrm{mK}$. We confirm that ${ }^{4} \mathrm{He}_{2} \mathrm{~A}$ clusters are bound. Our results are in excellent agreement with the latest adiabatic hyperspherical results from Ref. 15, which were obtained with potentials almost the same to the ones used here: the same KTTY interaction for He-A and for He-He the latest interaction potential ${ }^{23}$ which, as we commented before, is extremely close to HFDB potential. The results from Ref. 11, 
obtained also by the DMC method, predict slightly weaker binding, which is understandable because they use the same He-A potential and a slightly weaker He-He model. The agreement between DMC and other state-of-the-art methods in the prediction of the ground-state properties of weakly bound systems has been also previously observed, for example in the study of ${ }^{4} \mathrm{He}_{2}{ }^{3} \mathrm{He}$ and ${ }^{4} \mathrm{He}_{3}$, as compared in detail in Ref. 19.

In the case of ${ }^{3} \mathrm{He}^{4} \mathrm{HeA}$ trimers, bound states are also obtained for all the considered alkali atoms A. They are all very weakly bound, for ${ }^{6} \mathrm{Li},{ }^{7} \mathrm{Li}$ and ${ }^{133} \mathrm{Cs}$ even less than quantum halo state ${ }^{4} \mathrm{He}_{2}{ }^{3} \mathrm{He}$, whose energy is predicted to be $-17.07(15)$ for $\mathrm{HFDB}^{19}$. In order to verify the sensitivity to the interaction potential models we have performed the calculations of ${ }^{3} \mathrm{He}^{4} \mathrm{He}^{7} \mathrm{Li},{ }^{3} \mathrm{He}^{4} \mathrm{He}^{23} \mathrm{Na}$ and ${ }^{3} \mathrm{He}^{4} \mathrm{He}^{39} \mathrm{~K}$ also using other He-A potentials. In the case of the XKMX potential quite good agreement is obtained for the ${ }^{3} \mathrm{He}^{4} \mathrm{He}^{23} \mathrm{Na}$, which is expected because XKMX and KTTY were constructed by fitting the same data. Similar agreement is observed for ${ }^{4} \mathrm{He}_{2}{ }^{23} \mathrm{Na}$. However, sizable differences appear in the ${ }^{3} \mathrm{He}^{4} \mathrm{He}^{7} \mathrm{Li}$, because even small differences in potential energy surface can be dramatically reflected in extremely weakly bound few-body systems. On first sight negligible discrepancy of fit in construction of XKMX causes in this case three times weaker binding than predicted with the original KTTY data. Approximately two times stronger binding than with KTTY is obtained when using the PSL potential, which is expected because it predicts significantly deeper potential energy surface than other models. In the Cvetko case, weaker binding is predicted in the case of ${ }^{3} \mathrm{He}^{4} \mathrm{He}{ }^{7} \mathrm{Li}$, but stronger for ${ }^{3} \mathrm{He}^{4} \mathrm{He}^{39} \mathrm{~K}$ which can be easily understood by inspecting the two interaction potential models (e.g. for He-K in Fig. 1).

There are not many results in the literature to compare with. Yuan and $\operatorname{Lin}^{7}$, who use the KTTY model for all interactions predict no binding for ${ }^{3} \mathrm{He}^{4} \mathrm{He}^{6} \mathrm{Li}$ and weaker binding for ${ }^{3} \mathrm{He}^{4} \mathrm{He}^{7} \mathrm{Li}$ and ${ }^{3} \mathrm{He}^{4} \mathrm{He}^{23} \mathrm{Na}$. However, their predictions for ${ }^{4} \mathrm{He}_{2} \mathrm{~A}$ trimers is in disagreement with both the latest results from Suno ${ }^{15}$ and our predictions. We believe that the disagreement is attributable to their use of the KTTY model for the He-He interaction and the use of the older version of the dispersion coefficients in the KTTY model for He$\mathrm{Li}^{24}$. This can be seen also from the data on binding energy of the dimers, where we get disagreement for ${ }^{4} \mathrm{He}_{2}$ and ${ }^{4} \mathrm{HeLi}$, but not for ${ }^{4} \mathrm{HeNa}$. Ground-state energies for ${ }^{4} \mathrm{He}_{2},{ }^{4} \mathrm{He}-$ ${ }^{6} \mathrm{Li},{ }^{4} \mathrm{He}-{ }^{7} \mathrm{Li},{ }^{4} \mathrm{He}-{ }^{23} \mathrm{Na}$, and ${ }^{4} \mathrm{He}-{ }^{23} \mathrm{Na}$, from Ref. ${ }^{7}$ are respectively: $-1.31 \mathrm{mK},-0.12 \mathrm{mK},-2.16$ $\mathrm{mK},-28.98 \mathrm{mK}$ and $-1.24 \mathrm{mK}$. In the same order, we get: $-1.69 \mathrm{mK},-1.515 \mathrm{mK},-5.622 \mathrm{mK}$, $-28.98 \mathrm{mK}$ and $-1.242 \mathrm{mK}$. Our results are also in excellent agreement with dimer energies 
TABLE I. Binding energy $E_{3}$ of mixed helium-alkali trimers obtained for $\mathrm{KKTY}^{5}, \mathrm{XKMX}^{26}, \mathrm{PSL}^{27}$ and Cvetko ${ }^{29}$ models of potential $V$ in this work and compared to results given in References. The values in parenthesis are the errorbars of the DMC calculation, i.e. the uncertainty of the last digit in binding energy. The symbol - stands for unbound clusters. In Ref. 12 and 14 two numbers correspond to lower and upper bound to the energy.

\begin{tabular}{|c|c|c|c|c|c|c|c|c|c|}
\hline \multirow[b]{2}{*}{ Cluster } & \multicolumn{9}{|c|}{$-E_{3} / \mathrm{mK}$} \\
\hline & KTTY & XKMX & PSL & Cvetko & {$[7]^{a}$} & {$[11]^{b}$} & {$[12]^{c}$} & {$[14]^{c}$} & {$[15]^{d}$} \\
\hline${ }^{3} \mathrm{He}_{2}{ }^{23} \mathrm{Na}$ & $9.1(3)$ & & & $16.5(2)$ & 5.7 & & & & \\
\hline${ }^{3} \mathrm{He}_{2}{ }^{41} \mathrm{~K}$ & - & & & $22.4(2)$ & & & & & \\
\hline${ }^{3} \mathrm{He}_{2}{ }^{85} \mathrm{Rb}$ & - & & & $49.0(3)$ & & & & $40.8,38.6$ & \\
\hline${ }^{3} \mathrm{He}^{4} \mathrm{He}^{6} \mathrm{Li}$ & $5.9(2)$ & & & & - & & & & \\
\hline${ }^{3} \mathrm{He}^{4} \mathrm{He}^{7} \mathrm{Li}$ & $16.3(3)$ & $5.3(3)$ & $35.1(5)$ & $9.2(1)$ & 2.2 & & & & \\
\hline${ }^{3} \mathrm{He}^{4} \mathrm{He}^{23} \mathrm{Na}$ & $59.1(1)$ & $55.8(4)$ & $155.9(6)$ & & 18.3 & & & & \\
\hline${ }^{3} \mathrm{He}^{4} \mathrm{He}^{39} \mathrm{~K}$ & $22.9(1)$ & & & $76.0(2)$ & & & $42.4,11$ & & \\
\hline${ }^{3} \mathrm{He}^{4} \mathrm{He}^{41} \mathrm{~K}$ & $23.7(2)$ & & & & & & & & \\
\hline${ }^{3} \mathrm{He}^{4} \mathrm{He}^{85} \mathrm{Rb}$ & $20.6(2)$ & & & & & & & $69.0,65.9$ & \\
\hline${ }^{3} \mathrm{He}^{4} \mathrm{He}^{133} \mathrm{Cs}$ & $9.7(2)$ & & & & & & & & \\
\hline${ }^{4} \mathrm{He}_{2}{ }^{6} \mathrm{Li}$ & $58.7(2)$ & & & & 31.4 & & & & 58.88 \\
\hline${ }^{4} \mathrm{He}_{2}{ }^{7} \mathrm{Li}$ & $81.0(3)$ & $57.6(3)$ & $113.1(4)$ & & 45.7 & 80.0 & & & 81.29 \\
\hline${ }^{4} \mathrm{He}_{2}{ }^{23} \mathrm{Na}$ & $152.7(1)$ & $147.6(4)$ & $286.7(8)$ & & 103.1 & 148.5 & & & 152.68 \\
\hline${ }^{4} \mathrm{He}_{2}{ }^{39} \mathrm{~K}$ & $89.7(2)$ & & & & & & $115,66.6$ & & 89.76 \\
\hline${ }^{4} \mathrm{He}_{2}{ }^{41} \mathrm{~K}$ & $91.2(2)$ & & & & & & & & 91.12 \\
\hline${ }^{4} \mathrm{He}_{2}{ }^{85} \mathrm{Rb}$ & $84.9(2)$ & & & & & & & $155 ., 152$. & 84.69 \\
\hline${ }^{4} \mathrm{He}_{2}{ }^{133} \mathrm{Cs}$ & $61.7(2)$ & & & & & & & & 61.77 \\
\hline
\end{tabular}

${ }^{a}$ Using the He-A and He-He potential from Ref. 24

${ }^{b}$ Using the He-A potential from Ref. 5 and He-He potential from Ref. 32

${ }^{c}$ Using the He-A potential from Ref. 24 and He-He potential from Ref. 31

${ }^{d}$ Using the He-A potential from Ref. 5 and He-He potential from Ref. 23.

from Refs. 5 and 16. 
Li, Gou and Shi ${ }^{12}$, who use similar potential models to ours give for the ${ }^{3} \mathrm{He}^{4} \mathrm{He}^{39} \mathrm{~K}$ only lower and upper bounds for the energy, which comprise also our prediction using the KTTY model. On the other hand, the prediction of $\mathrm{Li}$, Zhang and Gou ${ }^{14}$ for lower and upper bounds of energy, in the case of ${ }^{3} \mathrm{He}^{4} \mathrm{He}^{85} \mathrm{Rb}$, are much lower than our result. Although they use the original version of the KTTY potential ${ }^{24}$ and a slightly different He-He model ${ }^{31}$ we do not believe it can explain the difference. However, the disagreement is also present in the case of other trimers with $\mathrm{Rb}$, where in particular for ${ }^{4} \mathrm{He}_{2}{ }^{85} \mathrm{Rb}$ our DMC calculation agrees with the adiabatic hyperspherical representation used in Ref. 15.

Due to the smaller mass of ${ }^{3} \mathrm{He}$ only ${ }^{3} \mathrm{He}_{2}{ }^{23} \mathrm{Na}$ is bound, in the case of KTTY model. Since the Cvetko potential is deeper in the case of He-K and He-Rb, bound states are also obtained for the corresponding trimers. In the case of $\mathrm{He}-\mathrm{Na}$, the binding increases substantially (from -9.1(3) to $-16.5(2) \mathrm{mK}$ ) despite the fact that for sodium Cvetko and KTTY potentials are close. This is due to the fact that in these weakly bound systems there is a lot of cancellation between kinetic and potential energy so even tiny differences in interaction potentials have significant effect. As far as the comparison with results from the other authors is concerned, the situation is the same as in the case of ${ }^{3} \mathrm{He}^{4} \mathrm{HeA}$ trimers discussed previously.

The calculation of excited state energies is challenging for DMC because of the nodal constraint. However, for weakly bound states the energy of the excited Efimov state can be predicted from the ground state energies of dimers and trimers following the scale-independent approach introduced by Delfino et al. ${ }^{9}$. In Ref. 9, based on the data from Yuan and $\operatorname{Lin}^{7}$ it was predicted that ${ }^{4} \mathrm{He}_{2}{ }^{7} \mathrm{Li}$ had an excited state with the energy close to $-2.31 \mathrm{mK}$, while no Efimov state was found for ${ }^{4} \mathrm{He}_{2}{ }^{6} \mathrm{Li}$. With our present results for the dimer and trimer energies, and the plot of the scaling limit from Ref. 9 we get an excited state of $-1.72 \mathrm{mK}$ for the ${ }^{4} \mathrm{He}_{2}{ }^{6} \mathrm{Li}$, while the ratio of the dimer and trimer energies would be outside the scaling limit for ${ }^{4} \mathrm{He}_{2}{ }^{7} \mathrm{Li}$. On the other hand, Suno et al. ${ }^{15}$ obtained $-2.09 \mathrm{mK}$ for the ${ }^{4} \mathrm{He}_{2}{ }^{6} \mathrm{Li}$. Although the agreement with our prediction is not perfect, it shows the relevance of the ratios between the energies of the two-body subsystems and the three-body system, which is the basis of the scaling approach in Ref. 9. 


\section{B. Structural properties}

In addition to energy, we determined the structure of the trimers using pure estimators to remove any bias coming from the trial wave function used for the guided diffusion in DMC. The pair distribution functions of ${ }^{3} \mathrm{He}_{2}{ }^{23} \mathrm{Na}$ and ${ }^{4} \mathrm{He}_{2} \mathrm{~A}$ are presented in Fig. 2 and those of ${ }^{3} \mathrm{He}^{4} \mathrm{HeA}$ in Fig. 3. All the distributions are normalized to $\int P(r) \mathrm{d} r=1$.

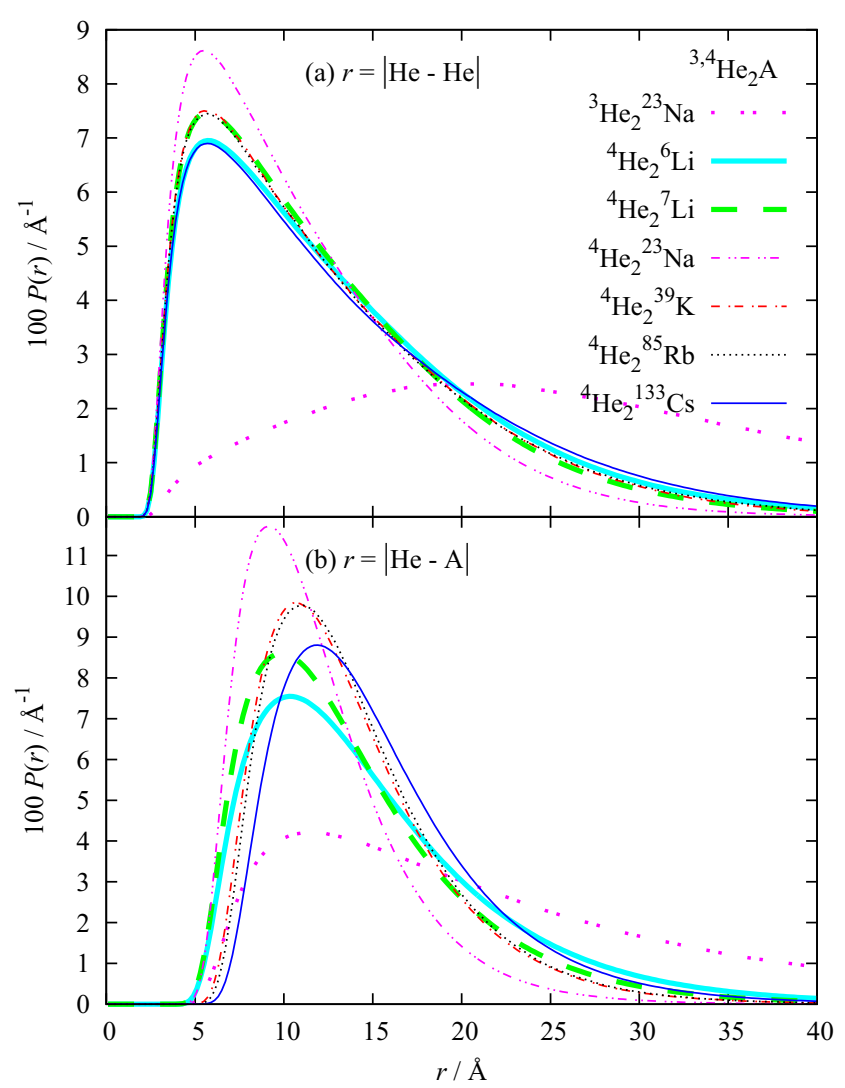

FIG. 2. Ground-state pair distributions $P(r)$ in clusters ${ }^{3} \mathrm{He}_{2}{ }^{23} \mathrm{Na}$ and ${ }^{4} \mathrm{He}_{2} \mathrm{~A}$, where A denotes an alkali metal, are shown as a function of separation $r$ between atoms: (a) He-He; (b) He-A. $P(r)$ are normalized to $\int P(r) \mathrm{d} r=1$.

We observe that ${ }^{4} \mathrm{He}$ atoms are on average closest in the case of the most strongly bound trimer ${ }^{4} \mathrm{He}_{2}{ }^{23} \mathrm{Na}$, whose $P(r)$ has the largest maximum and fastest decay. The trimers with similar binding energies have almost the same $P(r)$ of ${ }^{4} \mathrm{He}-{ }^{4} \mathrm{He}$ pair. Thus, they are very close for ${ }^{4} \mathrm{He}_{2}{ }^{7} \mathrm{Li},{ }^{4} \mathrm{He}_{2}{ }^{39} \mathrm{~K}$ and ${ }^{4} \mathrm{He}_{2}{ }^{85} \mathrm{Rb}$, as well as for ${ }^{4} \mathrm{He}_{2}{ }^{6} \mathrm{Li}$ and ${ }^{4} \mathrm{He}_{2}{ }^{133} \mathrm{Cs}$. However, more spread is observed in the case of $P(r)$ for the He-A pair, because the hard wall determines the correlation hole at small distances, which grows from Li to Cs. On the other hand, long 


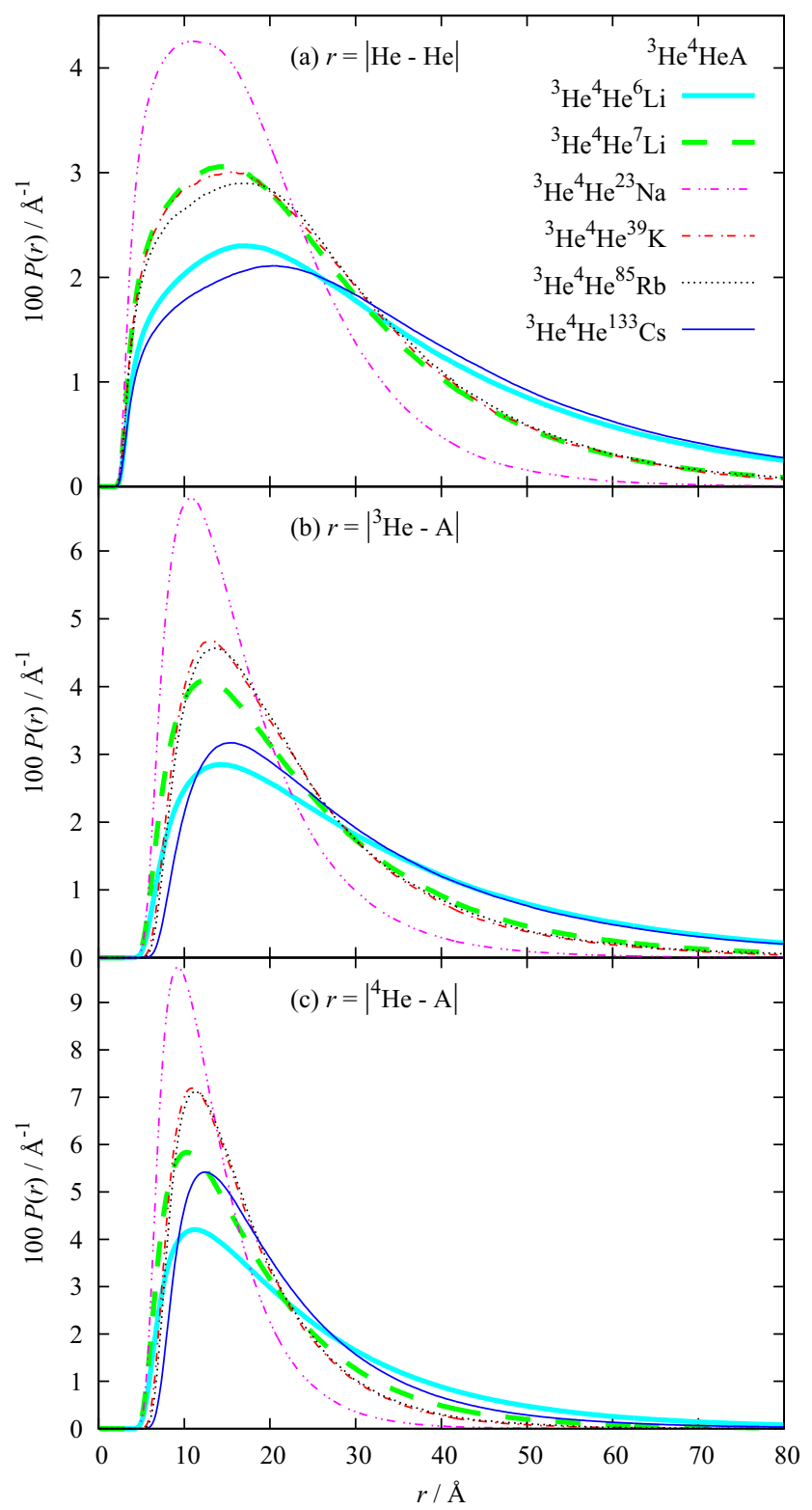

FIG. 3. Ground-state pair distributions $P(r)$ in clusters ${ }^{3} \mathrm{He}^{4} \mathrm{HeA}$, where A denotes an alkali metal, are shown as a function of separation $r$ between atoms: (a) ${ }^{3} \mathrm{He}-{ }^{4} \mathrm{He}$; (b) ${ }^{3} \mathrm{He}-\mathrm{A}$; (c) ${ }^{4} \mathrm{He}-\mathrm{A} . \mathrm{P}(r)$ are normalized to $\int P(r) \mathrm{d} r=1$.

range decay is faster for more strongly bound pairs. The most striking feature in the Fig. 2 is the huge size of ${ }^{3} \mathrm{He}_{2}{ }^{23} \mathrm{Na}$ pair distribution functions compared to all the others with two ${ }^{4} \mathrm{He}$ atoms. It is a consequence of one order of magnitude weaker binding of the trimer and is consistent with its predicted quantum halo character. ${ }^{17}$

Fig. 3 presents distribution functions for three different pairs in each trimer ${ }^{3} \mathrm{He}^{4} \mathrm{HeA}$. 
Not surprisingly, on average, ${ }^{4} \mathrm{He}$ is closer to the $A$ atom than ${ }^{3} \mathrm{He}$. The highest maxima are again observed for the most strongly bound trimer, the one with Na. All distributions are however very wide and particles have huge probability to be found in the classically forbidden region. In Ref. 17, several of those trimers were predicted, just like ${ }^{3} \mathrm{He}_{2}{ }^{23} \mathrm{Na}$, to be quantum halo states.

More insight into the structure of the trimers can be obtained by looking at the density profiles with respect to the center of mass, shown in Figs. 4 and 5. All the distributions are normalized to 1 as $4 \pi \int \rho(r) r^{2} \mathrm{~d} r=1$. In all trimers there is a reduced probability to find He atoms in the center of mass. The stonger the binding, the faster is the decay of He distributions at long distances, as can be best seen in trimers containing both ${ }^{4} \mathrm{He}$ and ${ }^{23} \mathrm{Na}$. With the increase of A mass, He atoms are pushed from the center so that, e.g. in trimers with ${ }^{133} \mathrm{Cs}$ atoms, He atoms have basically zero probability to be found closer than $5 \AA$ to the center of mass. At the same time, Cs is not likely to be found more than $2 \AA$ away from the center of mass. The density distribution function of alkali atoms expectedly becomes wider with the decrease of the isotope mass. Due to the trimer floppy nature, the atoms assume many different configurations. This is reflected in the two-peaked structure of the A density profiles in Fig. 5.

Possible shapes of triangular configurations can be studied by calculating angular distributions, $P(\vartheta)$. They are normalized as $\int P(\vartheta) \mathrm{d} \vartheta=1$. The results for trimers with the same two helium isotopes are presented in Fig. 6. The distributions are very wide, confirming the trimers' floppy nature and indicating no preferred shape. All ${ }^{4} \mathrm{He}_{2} \mathrm{~A}$ trimers have very similar distributions, which is understandable since they are all of all-bound type, that is all dimer pairs are bound. Significantly different shapes are obtained for the ${ }^{3} \mathrm{He}_{2}{ }^{23} \mathrm{Na}$ trimer, which is a quantum halo system and thus much more spatially extended. It is of samba type, ${ }^{34}$ which means that ${ }^{3} \mathrm{He}^{23} \mathrm{Na}$ form two bound pairs, while two ${ }^{3} \mathrm{He}$ atoms are unbound. The alkali atom is typically very near the centre of mass. The distribution of the angle which has the alkali atom at the vertex is peaked around 20 degrees for ${ }^{4} \mathrm{He}_{2} \mathrm{~A}$ trimers, while for ${ }^{3} \mathrm{He}_{2}{ }^{23} \mathrm{Na}$ it is much more spread with significantly more weight at larger angles. At the same time, $P(\vartheta)$ for the angles with He atoms at the vertices has more weight at lower angles for ${ }^{3} \mathrm{He}_{2}{ }^{23} \mathrm{Na}$ than for ${ }^{4} \mathrm{He}_{2} \mathrm{~A}$ trimers. This is consistent with ${ }^{23} \mathrm{Na}$ having significantly more scalene configurations, where ${ }^{23} \mathrm{Na}$ is at the center of mass and ${ }^{3} \mathrm{He}$ atoms tend to be further apart. 


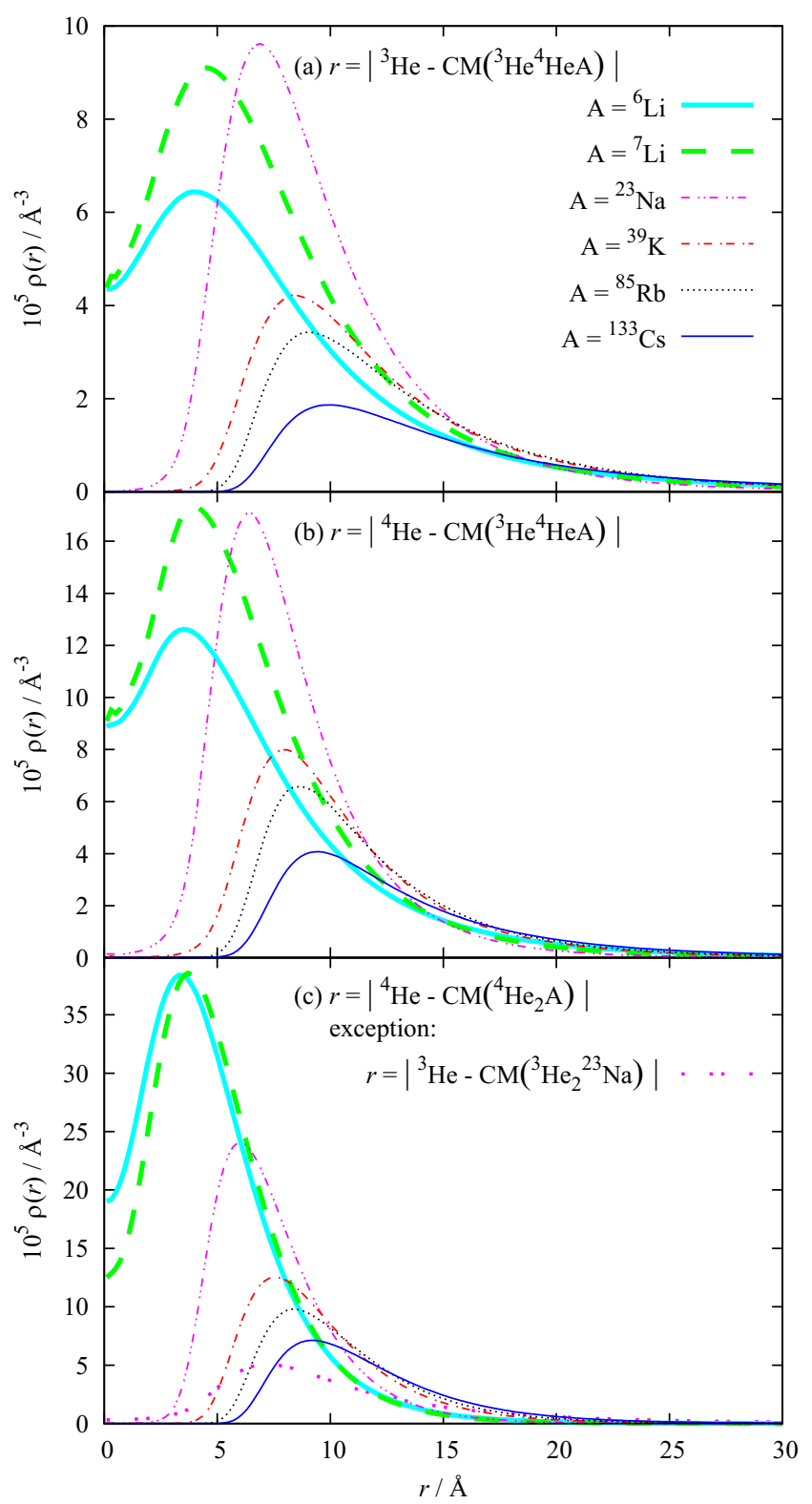

FIG. 4. Ground-state density profiles $\rho(r)$ with respect to the center of mass $(\mathrm{CM})$ : (a) ${ }^{3} \mathrm{He}$ and (b) ${ }^{4} \mathrm{He}$ in ${ }^{3} \mathrm{He}^{4} \mathrm{HeA}$; (c) He in ${ }^{4} \mathrm{He}_{2} \mathrm{~A}$ and ${ }^{3} \mathrm{He}_{2}{ }^{23} \mathrm{Na}$. Different line types distinguish alkali metals (A). $\rho(r)$ are normalized to $4 \pi \int \rho(r) r^{2} \mathrm{~d} r=1$.

Angular distribution functions for ${ }^{3} \mathrm{He}^{4} \mathrm{HeA}$ trimers are reported in Fig. 7 for all three angles. The widest distributions are obtained for the angles centered at the alkali atom, which is always, as discussed previously, close to the center. These trimers belong to the different types of clusters. ${ }^{3} \mathrm{He}^{4} \mathrm{He}^{6} \mathrm{Li},{ }^{3} \mathrm{He}^{4} \mathrm{He}^{7} \mathrm{Li},{ }^{3} \mathrm{He}^{4} \mathrm{He}^{39} \mathrm{~K}$, and ${ }^{3} \mathrm{He}^{4} \mathrm{He}^{85} \mathrm{Rb}$ are tango states ${ }^{35}$ because only the ${ }^{4} \mathrm{He}-\mathrm{A}$ pair is bound, while ${ }^{3} \mathrm{He}^{4} \mathrm{He}^{23} \mathrm{Na}$ is samba because both 


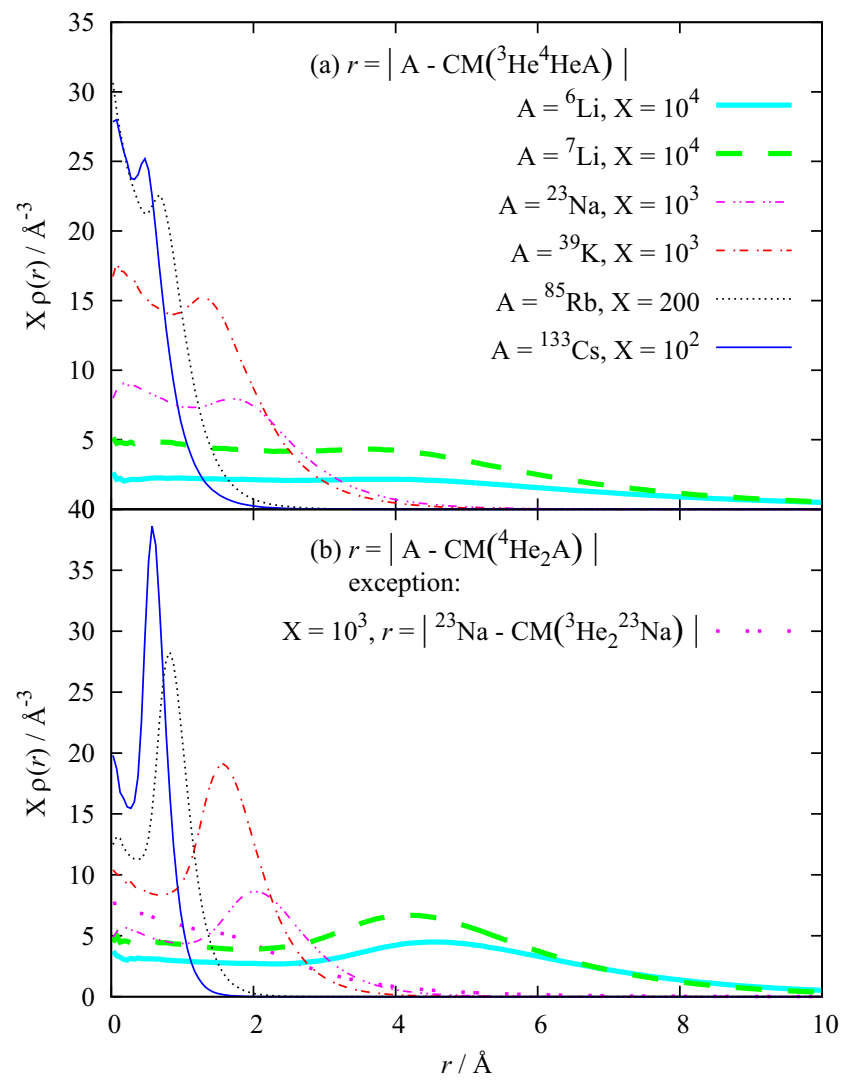

FIG. 5. Ground-state density profiles $\rho(r)$ with respect to the center of mass (CM) for alkali metals (A) distinguished by different line types in: (a) ${ }^{3} \mathrm{He}^{4} \mathrm{HeA}$; (b) ${ }^{4} \mathrm{He}_{2} \mathrm{~A}$ and ${ }^{3} \mathrm{He}_{2}{ }^{23} \mathrm{Na} . \quad \rho(r)$ are normalized to $4 \pi \int \rho(r) r^{2} \mathrm{~d} r=1$ and multiplied by a given factor $\mathrm{X}$ for visible comparison.

${ }^{3} \mathrm{He}^{23} \mathrm{Na}$ and ${ }^{4} \mathrm{He}-{ }^{23} \mathrm{Na}$ form a bound state. However, the angular distributions for different A isotopes differ more than in the case of ${ }^{4} \mathrm{He}_{2} \mathrm{~A}$ trimers. It can be understood by inspecting their scaled sizes and energies. In Ref. 19 it was shown that ${ }^{3} \mathrm{He}^{4} \mathrm{He}^{7} \mathrm{Li},{ }^{3} \mathrm{He}^{4} \mathrm{He}^{39} \mathrm{~K}$ and ${ }^{3} \mathrm{He}_{2}{ }^{23} \mathrm{Na}$ are not close on the scaling plot, thus it can not be expected that they have very similar distributions of trimer configurations.

\section{SUMMARY AND CONCLUSIONS}

We have obtained ground state energy and structural properties of trimers consisting of one alkali and two helium atoms, using accurate diffusion Monte Carlo simulations.

Our predictions for the ground-state energy are in excellent agreement with the most recent estimates of ${ }^{4} \mathrm{He}_{2} \mathrm{~A}$ trimers, obtained with different methods, confirming their accuracy. 


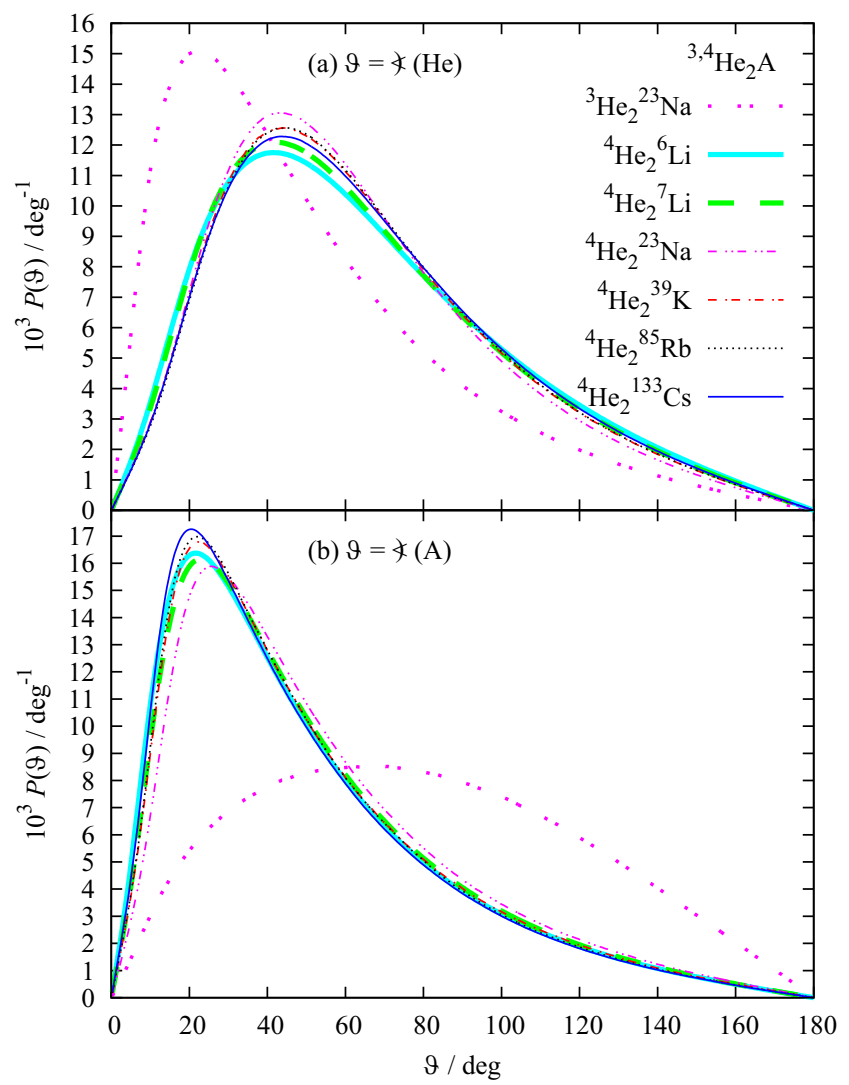

FIG. 6. Ground-state angular distributions $P(\vartheta)$ in trimers ${ }^{3} \mathrm{He}_{2}{ }^{23} \mathrm{Na}$ and ${ }^{4} \mathrm{He}_{2} \mathrm{~A}$, where A denotes an alkali metal, are shown as a function of corner angle $\vartheta$ : (a) $\measuredangle(\mathrm{He}-\mathrm{He}-\mathrm{A})$; (b) $\measuredangle(\mathrm{He}-\mathrm{A}-\mathrm{He}) . P(\vartheta)$ are normalized to $\int P(\vartheta) \mathrm{d} \vartheta=1$.

For the trimers ${ }^{3} \mathrm{He}^{4} \mathrm{HeA}$ and ${ }^{3} \mathrm{He}_{2} \mathrm{~A}$, our calculation gives the first precise estimate using the latest He-A interaction potentials. Furthermore, we predict for the first time the bound state of ${ }^{3} \mathrm{He}^{4} \mathrm{He}^{6} \mathrm{Li}$ and ${ }^{3} \mathrm{He}^{4} \mathrm{He}^{133} \mathrm{Cs}$. Our results on the stability of the trimers depend sensitively on the interaction potential model. Thus, changing from the KTTY model to Cvetko one, we also get that ${ }^{3} \mathrm{He}_{2}{ }^{41} \mathrm{~K}$ and ${ }^{3} \mathrm{He}_{2}{ }^{85} \mathrm{Rb}$ are bound.

Structural properties for most trimers were obtained here for the first time. Very low binding energies and large spatial extent for most of the molecules with ${ }^{3} \mathrm{He}$, suggest their quantum halo nature. Although there are no experimental measurements to compare the results with, the measurement of the helium trimers' distribution functions bring optimism that such a feat might be achieved in He-alkali systems as well. It would be extremely useful for testing the interaction potentials between helium and alkali atoms, which are not as well known as the He-He interaction potential. 


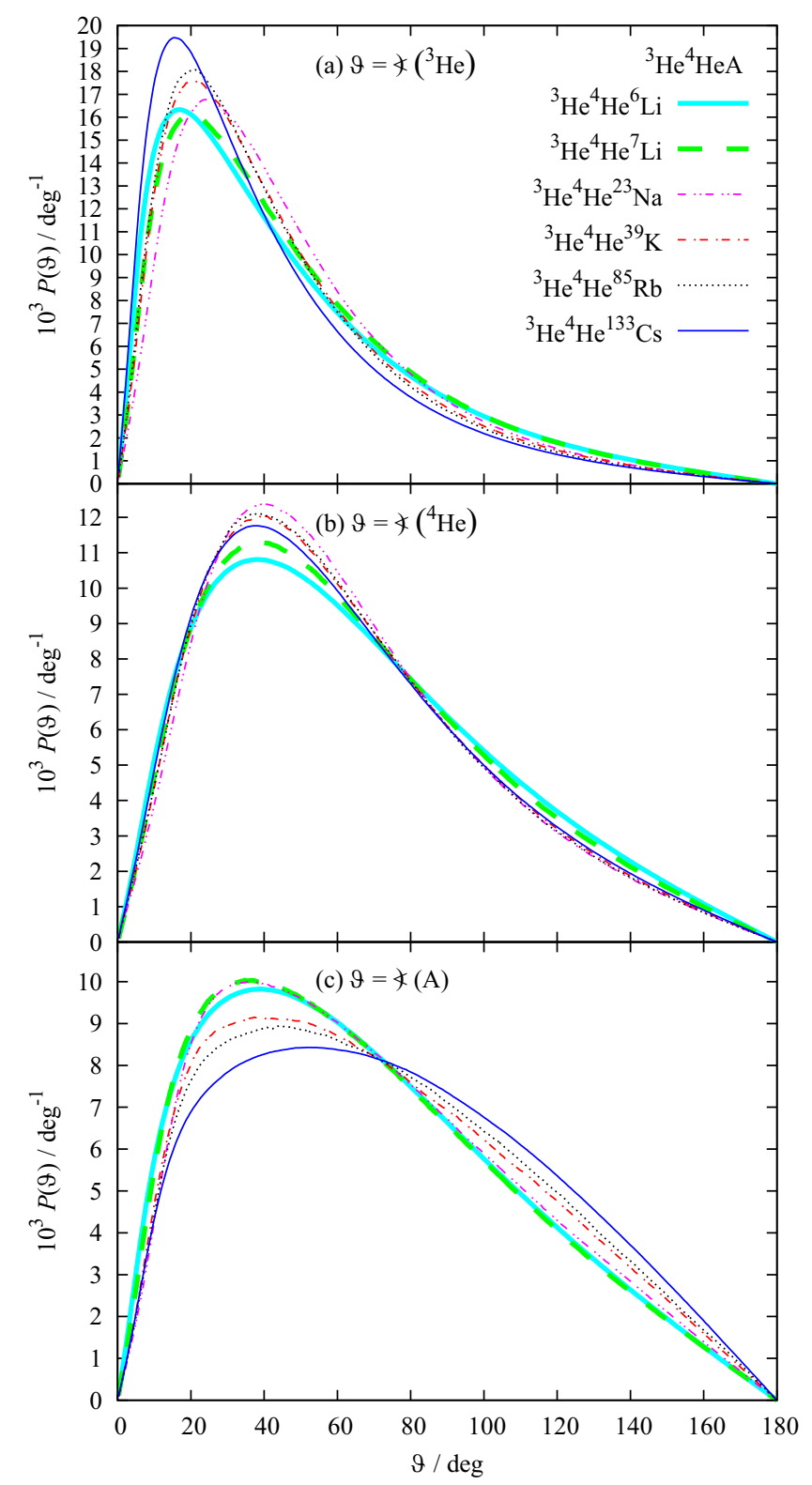

FIG. 7. Ground-state angular distributions $P(\vartheta)$ in trimers ${ }^{3} \mathrm{He}^{4} \mathrm{HeA}$, where A denotes an alkali metal, are shown as a function of corner angle $\vartheta$ : (a) $\measuredangle\left(\mathrm{A}-{ }^{3} \mathrm{He}-{ }^{4} \mathrm{He}\right)$; (b) $\measuredangle\left({ }^{3} \mathrm{He}-{ }^{4} \mathrm{He}-\mathrm{A}\right)$; (c) $\measuredangle\left({ }^{4} \mathrm{He}-\mathrm{A}-{ }^{3} \mathrm{He}\right) . P(\vartheta)$ are normalized to $\int P(\vartheta) \mathrm{d} \vartheta=1$.

\section{ACKNOWLEDGMENTS}

This work has been supported in part by the Croatian Science Foundation under the project number IP-2014-09-2452. J. B. acknowledge additional support by the MICINNSpain, Grant No. FIS2014-56257-C2-1-P. The computational resources of the Isabella cluster at Zagreb University Computing Center (Srce), the HYBRID cluster at the University of 
Split, Faculty of Science and Croatian National Grid Infrastructure (CRO NGI) were used.

\section{REFERENCES}

${ }^{1}$ A. S. Jensen, K. Riisager, D. V. Fedorov, and E. Garrido, Rev. Mod. Phys. 76, 215 (2004).

${ }^{2}$ K. Riisager, Phys. Scr. T152, 014001 (2013).

${ }^{3}$ V. Efimov, Phys. Lett. 33B, 563 (1970).

${ }^{4}$ Maksim Kunitski, Stefan Zeller, Jörg Voigtsberger, Anton Kalinin, Lothar Ph. H. Schmidt, Markus Schöffler, Achim Czasch, Wieland Schöllkopf, Robert E. Grisenti, Till Jahnke, Dörte Blume, and Reinhard Dörner, Science 348, 551 (2015).

${ }^{5}$ U. Kleinekathofer, M. Lewerenz, and M. Mladenovic, Phys. Rev. Lett. 83, 4717 (1999).

${ }^{6}$ N. Tariq, N. A. Taisan, V. Singh, and J. D. Weinstein, Phys. Rev. Lett. 110, 153201 (2013).

${ }^{7}$ J. Yuan and C. D. Lin, J. Phys. B 31, L637 (1998).

${ }^{8}$ I. Baccarelli, G. Delgado-Barrio, F. A. Gianturco, T. Gonzalez-Lezana, S. Miret-Artes and P. Villarreal, Phys. Chem. Chem. Phys., 2, 4067 (2000).

${ }^{9}$ A. Delfino, T. Frederico, and L. Tomio, J. Chem. Phys. 113, 7874 (2000).

${ }^{10}$ I. Baccarelli, G. Delgado, F. A. Gianturco, T. Gonzalez-Lezana, S. Miret-Artes and P. Villarreal, Europhys. Lett. 50, 567 (2000).

${ }^{11}$ C. DiPaola, F. A. Gianturco, F. Paesani, G. Delgado BarrioT. Gonzales-Lezana, S. MiretArtes, and P. Villarreal, I. Baccarelli, and T. Gonzales-Lezana, J. Phys. B 85, 2643 (2002). ${ }^{12}$ Y. Li, Q. Gou, and T. Shi, Phys. Rev. A 74, 032502 (2006).

${ }^{13}$ H. Suno, B. D. Esry, Phys. Rev. A 82, 062521 (2010).

${ }^{14}$ Y. Li, W. Zhang, Q. Gou, H. Song, and T. Shi, Phys. Rev. A 82, 022515 (2010).

${ }^{15}$ H. Suno, E. Hiyama and M. Kamimura, Few Body Systems 54, 1557 (2013).

${ }^{16}$ H. Suno, B. D. Esry, Phys. Rev. A 89, 052701 (2014).

${ }^{17}$ P. Stipanović, L. Vranješ Markić, I. Bešlić, and J. Boronat, Phys. Rev. Lett. 113, 253401 (2014).

${ }^{18}$ J. Voigtsberger, S. Zeller, J. Becht, N. Neumann, F. Sturm, H-K. Kim, M. Waitz, F. Trinter, M. Kunitski, A. Kalinin, J. Wu, W. Schöllkopf, D. Bressanini, A. Czasch, J. B. Williams, K. Ullmann-Pfleger, L. Ph H. Schmidt, M. S. Schöffler, R. E. Grisenti, T. Jahnke, and R. Dörner, Nature Communications 5, 5765 (2014). 
${ }^{19}$ P. Stipanović, L. Vranješ Markić, and J. Boronat, J. Phys. B: At. Mol. Opt. Phys. 49, 185101 (2016).

${ }^{20}$ J. Boronat and J. Casulleras, Phys. Rev. B 49, 8920 (1994).

${ }^{21}$ J. Casulleras and J. Boronat, Phys. Rev. B 52, 3654 (1995).

${ }^{22}$ R. A. Aziz, F. R. W. McCourt, and C. C. K. Wong, Mol. Phys. 61, 1487 (1987).

${ }^{23}$ M. Jeziorska, W. Cencek, B. Patkowski, B. Jeziorski, and K. Szalewicz, J. Chem. Phys. 127, 124303 (2007).

${ }^{24}$ U. Kleinekathofer, K. T. Tang, J. P. Toennies, and C. L. Yiu, Chem. Phys. Lett. 249, 257 (1996).

${ }^{25}$ Zong-Chao Yan, James F. Babb, A. Dalgarno, and G. W. F. Drake, Phys. Rev A 54, 2824(1996).

${ }^{26}$ J. C. Xie, T. Kar, S. K. Mishra, R-H. Xie, Chem. Phys. Lett., 591, 69 (2014).

${ }^{27}$ H. Partridge, J. R. Stallcop, and E. Levin, J. Chem. Phys. 115, 6471 (2001).

${ }^{28}$ D. Spelsberg, T. Lorenz, and W. Meyer, J. Chem. Phys. 99, 7845 (1993).

${ }^{29}$ D. Cvetko, A. Lausi, A. Morgante, F. Tommasini, P. Cortona, and M. G. Dondi, J. Chem. Phys. 100, 2052 (1994).

${ }^{30}$ H. Suno, B. D. Esry, Phys. Rev. A 78, 062701 (2008).

${ }^{31}$ R. A. Aziz and M. J. Slaman, J. Chem. Phys. 94, 8047 (1991).

${ }^{32}$ K. T. Tang, J. P. Toennies and C. L. Yiu, Phys. Rev. Lett. 74, 4571 (1995).

${ }^{33}$ V. Roudnev and M. Cavagnero, J. Phys. B: At. Mol. Opt. Phys. 45, 025101, (2012).

${ }^{34}$ M. T. Yamashita, L. Tomio, T. Frederico, Nuclear Phys. A 735, 40 (2004).

${ }^{35}$ F. Robicheaux, Phys. Rev. A 60, 1706 (1999). 